A N N A L E S Annales de Bretagne et des Pays de l'Ouest

Anjou. Maine. Poitou-Charente. Touraine

113-1 | 2006

Varia

\title{
Le mariage mixte au XVIIIe siècle
}

\section{Sébastien Jahan}

\section{OpenEdition}

\section{Journals}

Édition électronique

URL : http://journals.openedition.org/abpo/900

DOI : 10.4000/abpo.900

ISBN : 978-2-7535-1501-7

ISSN : 2108-6443

Éditeur

Presses universitaires de Rennes

Édition imprimée

Date de publication : 30 mars 2006

Pagination : $53-70$

ISBN : 978-2-7535-0273-4

ISSN : 0399-0826

Référence électronique

Sébastien Jahan, «Le mariage mixte au XVIIIe siècle », Annales de Bretagne et des Pays de l'Ouest [En ligne], 113-1| 2006, mis en ligne le 30 mars 2008, consulté le 20 avril 2019. URL : http:// journals.openedition.org/abpo/900 ; DOI : 10.4000/abpo.900 


\title{
Le mariage mixte au XVIII ${ }^{\mathrm{e}}$ siècle
}

\author{
Sébastien JaHAN \\ Maître de conférences en histoire moderne \\ GERHICO - université de Poitiers
}

Apparue dans la première moitié du xxe siècle, l'expression " mariage mixte " ne fait pas partie de l'arsenal conceptuel classique de l'historien. Ce sont plutôt les sociologues et les géographes qui ont mis cette formule à l'honneur dans leurs études sur l'immigration et la communication entre les cultures. Même s'ils privilégient parfois un autre vocabulaire (inter mariage, mariage exogame français-étranger, cross-cultural ou interethnic marriage chez les Anglo-Saxons...), ces travaux viennent alimenter l'idée que l'échange matrimonial serait l'aboutissement inéluctable de la migration, une étape essentielle dans le processus de dissolution de la spécificité culturelle dans la société réceptrice, voire le signe le plus probant de la volonté des étrangers de faire partie intégrante de leur milieu d'accueil ${ }^{1}$. En France et dans de nombreux autres pays européens, la soi-disant moindre propension des Maghrébins - et d'une façon générale des immigrés de confession musulmane - à l'inter mariage a, du coup, un temps contribué à jeter l'opprobre sur une population soupçonnée de rejeter le modèle culturel de leurs hôtes et " bienfaiteurs ${ }^{2}$ ". On peut aussi penser que le test pour être significatif n'est peut-être pas exclusif et que d'autres indicateurs sont tout aussi aptes à mettre en évidence l'intégration (la pratique religieuse, le parcours professionnel, etc.). Sans nier l'existence et le poids des prescriptions islamiques régissant le mariage, il n'est surtout pas inutile de rappeler ici que le couple mixte franco-maghrébin ou franco-afri-

1. «L'échange des femmes est, lorsque deux groupes humains entrent en contact, un mécanisme anthropologique fondamental : s'il se produit, il implique une dynamique d'assimilation, s'il est refusé, une trajectoire de ségrégation. Le taux d'exogamie, proportion de mariages réalisés par les immigrés, leurs enfants ou petits-enfants avec des membres de la société d'accueil, est l'indicateur anthropologique ultime d'assimilation ou de ségrégation, qui peut opposer sa vérité à celle des indicateurs politiques et idéologiques " (ToDd, Emmanuel, Le Destin des immigrés. Assimilation et ségrégation dans les démocraties occidentales, Paris, Le Seuil, 1994, p. 10-11).

2. Emmanuel Todd (ibidem, p. 305-306) montre à rebours de ce cliché, l'augmentation rapide du taux d'exogamie chez les jeunes femmes algériennes alors même que leur mariage avec un non musulman est perçu en théorie comme " une infidélité à l'Islam et au principe patrilinéaire ". 
cain en général, pour se bâtir, a dû surmonter d'autres obstacles essentiels à la rencontre et à l'acceptation de l'autre : le racisme et la xénophobie des populations " de souche " au premier chef, mais aussi la tendance renforcée de l'État légiférant à faire peser la suspicion sur ces unions sous le prétexte de lutter contre l'immigration clandestine. Problématique éminemment actuelle, la question du mariage mixte comme lieu d'expérimentation de " la tolérance à la différence " ou " caisse de résonance des malentendus et des conflits interculturels ${ }^{3}$ " pourrait pourtant se conjuguer à tous les temps du passé.

Dans la France d'Ancien Régime, le poids du christianisme et la dimension éminemment politique des affaires religieuses, la rareté de la rencontre " interethnique ", le resserrement des horizons et des frontières mentales, rendent tout aussi passionnante une question du mariage mixte qui ne saurait être vue comme un phénomène nouveau. L'expression "mariage mixte " véhicule des significations et des représentations variables selon les époques et les contextes culturels qui ont souvent plus à voir avec l'imaginaire, voire le fantasme, qu'avec l'appréciation objective d'une situation donnée. Cette labilité est en fait le reflet de perceptions diverses du même et de l'autre, de l'autorisé et de l'interdit. Hier comme aujourd'hui, la mixité nous parle de la norme, de sa fermeté, de sa transgression. Elle nous renseigne sur le degré d'ouverture d'une société et sur la manière dont les populations en contact vivent, se représentent, composent et recomposent leur identité.

\section{Définitions d'hier et d'aujourd'hui}

Le Petit Robert de 1994, à l'entrée " mixte ", propose la définition suivante, censée rendre compte de l'usage et de la représentation dominants. Est mixte ce « qui est formé de plusieurs, de deux éléments de nature différente " : une " salade mixte ", une " cuisinière mixte ", un " mariage mixte ". Pour cette dernière expression, l'article du dictionnaire précise qu'il s'agit d'une union " entre deux personnes de religion, de race ou de nationalité différentes ». En 1968, le même ouvrage en proposait une signification beaucoup plus restreinte, limitant la mixité matrimoniale au domaine de la religion, plus particulièrement encore aux noces d'un catholique et d'un chrétien d'une autre Église (un protestant, un orthodoxe, etc.) ${ }^{4}$. L'absence de termes pour désigner le mariage d'un chrétien avec un juif ou un musulman est-elle liée à la rareté de ce cas de figure, voire à son caractère inconcevable? À moins plutôt qu'un tel assortiment interconfessionnel soit considéré comme second par rapport à la dimension interethnique qu'il suppose nécessairement. Le fait est que la définition de 1994, en élargissant consi-

3. STREIFF-FENART, Jocelyne, Les Couples franco-maghrébins en France, L'Harmattan, "Logiques sociales", 1989, p. 13.

4. VARro, Gabrielle, "Critique raisonnée de la notion de mixité ", dans PHILPPE, Claudine, VARRO, Gabrielle et NEYRAND, Gérard (dir.), Liberté, égalité, mixité... conjugales, Une sociologie du couple mixte, Paris, Anthropos, 1998, p. 6-7. 
dérablement le champ sémantique couvert par l'expression, s'agrégeait la seule forme de mixité réellement admise par l'usage juridique et administratif français contemporain : un enquêteur de l'INED ne prendra en considération que la seule différence de nationalité pour caractériser un mariage mixte, tout simplement parce que la loi en vigueur interdit d'utiliser des référents tels que l'origine ethnique ou l'appartenance religieuse ${ }^{5}$. Dans un recensement nominatif, la confession n'est jamais signalée et l'immigré naturalisé ou son descendant ne se distinguent que sur la base - aléatoire - de l'onomastique ou du pays de naissance. Cette sage précaution pose cependant de réels problèmes aux démographes et aux sociologues qui, du coup, ne peuvent disposer que de données sous-évaluant nécessairement le phénomène de l'inter mariage. La tentation est grande, dans un questionnaire sociologique, de réintroduire la distinction entre "Français de souche " et "Français par acquisition " ou " issus de l'immigration ", quitte à entériner dans le vocabulaire une réalité discriminante ou d'en réactiver le sentiment parfois douloureux dans les consciences ${ }^{6}$.

Trop de mixité ou pas assez de mixités? La vision d'une différence cantonnée à la nationalité se heurte au scepticisme de ceux qui refusent d'enfermer les individus dans ce genre de catégories toutes faites, non seulement parce que cette idée d'un mariage mixte introduit la notion stigmatisante de l'étranger mais aussi parce qu'elle est impuissante à rendre compte de l'histoire d'une humanité mixte par nature comme de la diffusion d'un phénomène en passe d'être totalement banalisé par le brassage des populations. L'emploi de l'expression subit aussi la critique de ceux qui, au nom de la complexité des représentations de l'identité individuelle et conjugale, la jugent bien trop restrictive par rapport à la gamme des mixités repérables au-delà de ces points de focalisation religieux, raciaux ou nationaux. De la même façon que deux personnes mariées de nationalités différentes ne se considèrent pas forcément mixtes, un couple réunissant deux ressortissants, de même souche et de même religion, d'un même pays pourra au contraire se définir comme tel en fonction de paramètres aussi divers qu'une langue régionale pratiquée, le milieu social, le mode de vie, l'histoire familiale ou l'existence d'un handicap supposant une culture différente (la surdité par exemple...). De fait, la négation de la mixité peut se faire au nom de deux points de vue d'apparence divergents, mais qui, à bien y repenser, partagent le même idéal humaniste. Le " militant " qui se rappelle que l'expression " mariage mixte " est née dans les années trente du $\mathrm{xx}^{\mathrm{e}}$ siècle, dans un contexte de forte xénophobie pointant du doigt le pillage des ressources matrimoniales de la patrie, refusera ce marquage de l'immigré et de ses choix. Le sentimental y lira l'emblème de l'Amour qui transcende les frontières et finira par s'étonner que de savants

5. Philippe, C. et VARro, G., " Remarques préliminaires ", dans Liberté, égalité, mixité..., op. cit., p. XVIII-XIX.

6. NOIRIEL, Gérard, Le Creuset français. Histoire de l'immigration XIXe-XXe siècle, Paris, Le Seuil, 2001, p. 9 . 
esprits fixent leur attention sur ce qui sépare les conjoints, alors même que s'ils se marient, on peut raisonnablement supposer qu'il y a beaucoup de plus de choses pour les rapprocher que pour les éloigner. Cette apparente naïveté peut gêner aux entournures les certitudes de ceux qui font de toute union mixte une union problématique. Aussi, la définition retenue par les sociologues qui étudient la question s'efforce-t-elle désormais de fonder la mixité non pas tant sur des critères " objectifs " que sur l'appréciation des acteurs et de leur entourage. La mixité caractériserait donc «toute union conjugale, légale ou de fait, impliquant deux personnes de sexe opposé appartenant ou ayant appartenu à des nations différentes ou devenues distinctes, adhérant ou ayant adhéré à des religions différentes, issues d'ethnies ou de races différentes, si [c'est nous qui soulignons] l'une ou plusieurs de ces différences actuelles sont ressenties par au moins l'un des partenaires, comme une source de difficultés légales, sociales, culturelles ou affectives, ou si elles provoquent une réaction sur l'environnement social ${ }^{7}$ ". Cette définition pourrait servir à l'historien des sociétés d'Ancien Régime, n'était son incapacité à sonder les coeurs du passé et les mémoires pour la plupart englouties, partant, à prendre l'entière mesure de l'incidence de la mixité dans la vie du couple et de sa famille. Cela ne veut évidemment pas dire que l'histoire n'a pas son mot à dire sur la question. Cela signifie simplement que son approche sera nécessairement spécifique. La rareté des témoignages directs ou indirects l'oblige ainsi à questionner de manière nouvelle des sources habituelles comme les registres paroissiaux ou les minutiers notariaux. On en verra quelques illustrations dans les pages qui suivent. Par ailleurs, et au préalable, le chercheur se trouve contraint de bien poser les limites de ce dont il entend parler.

La première difficulté est liée à l'inexistence de l'expression " mariage mixte " pour les périodes antérieures au xx siècle. Si l'on suit le Dictionnaire de Furetière, dans son édition de 1690, l'adjectif mixte est alors réservé à l'usage du savant et désigne un " corps composé des quatre éléments de différentes parties ou atomes". L'article poursuit en précisant que " tous les corps de la région élémentaire sont réputés mixtes composé des éléments qui ne se trouvent point tout purs en la nature ${ }^{8}$ ". Propre à une physique aristotélicienne en passe d'être submergée par la diffusion des découvertes et des théories de Newton, cette définition est bien sûr fort éloignée de nos préoccupations. Elle témoigne à tout le moins d'une représentation savante du monde où la mixité, assimilée à l'impur, n'en reste pas moins la règle dans la nature, la pureté étant confinée au monde des sphères célestes.

L'impureté essentielle des créatures terrestres n'empêche cependant pas la première d'entre elles, l'être humain, de se méfier de certains mélanges matrimoniaux. Mais - et c'est là une autre difficulté - à la diffé-

7. PhILIPPE, C., "Profils de couples mixtes : essai de typologie ", dans Migrations Société, vol. $3, \mathrm{n}^{\circ} 14,1991$.

8. FURETIERE, Antoine, Dictionnaire universel contenant généralement les mots français tant vieux que modernes..., La Haye-Rotterdam, 1690, tome 2, art. " Mixte ", p. 644. 
rence de nos contemporains, les Français des Temps modernes portent plutôt leur attention sur les dysymétries de " condition " ou d'" état ". La mésalliance est, en effet, une catégorie de la langue juridique d'Ancien Régime que les hommes de loi préfèrent d'ailleurs appeler " mariage inégal ". Cette discordance dans le statut social des époux, sans être formellement prohibée ${ }^{9}$, suscitait néanmoins, on le sait, la réprobation des autorités attentives à endiguer toute forme de brouillage des conditions. Elle n'en était pas moins courante, pour autant, associant non seulement des lignages nobles et roturiers, mais aussi des familles de fortune ou de notoriété différentes au sein de chacun de ces deux groupes. Nul étonnement, dès lors, à ce que de nombreux historiens se soient employés à étudier ces unions-là qui contrarient la vision classique d'un mariage abusivement conçu comme plus déterminé par la "règle homogamique " que par un impératif de complémentarité. Réfléchir sur la mésalliance permettait à ces chercheurs de repenser les catégories qui servent à structurer cette société fortement hiérarchisée, jaugeant leur porosité et leur souplesse ou au contraire leur étanchéité et leur rigidité ${ }^{10}$. La rareté de ce que nous rangeons aujourd'hui sous le vocable de mariages mixtes pourrait certes s'expliquer dans une France d'Ancien Régime en apparence moins habituée à la cohabitation des nations et des religions et peuplée de paysans, pour la plupart, fort sédentaires. L'uniformité et l'immobilité ont été pourtant davantage des rêves du pouvoir absolutiste que des réalités tangibles à l'échelle de la vie des villes et des villages. La mixité conjugale a donc bien existé tant dans le sens que lui donnent les dictionnaires des $\mathrm{xx}^{\mathrm{e}}$ et $\mathrm{XXI}^{\mathrm{e}}$ siècles que dans d'autres dimensions qui sont propres à l'époque qui nous intéresse.

\section{Les formes de la mixité matrimoniale sous l'Ancien Régime : mariages interconfessionnels et interethniques}

Pour bien comprendre ce que pourrait être une union mixte sous l'Ancien Régime, il n'est pas inutile de rappeler brièvement comment le droit français conçoit le mariage, avant les mutations de la législation révolutionnaire et du Code Civil. Le mariage a une définition juridique civile et canonique indissociable. Il s'agit, en effet, d'un " contrat civil élevé à la dignité de Sacrement ". En tant que contrat civil, il suppose le consentement des conjoints et la conformité aux lois du Royaume. En tant que sacrement, il est indissoluble et béni par un prêtre. Pour pouvoir se marier, les

9. « L'inégalité des conditions n'est pas un moyen suffisant pour faire déclarer nul un mariage d'ailleurs valablement contracté " (FERRIERE, Claude-Joseph de, Dictionnaire de Droit et de Pratique, tome 2, Paris, 1768, art. "Mariage inégal ", p. 202). L'article précise cependant ensuite que l'Ordonnance de Blois « défend aux veuves ayant des enfants d'un autre mariage, qui se remarient à des personnes indignes, ou à leurs valets, de faire aucun avantage à leur second mari ".

10. Voir par exemple NASSIET, Michel, Noblesse et pauvreté. La petite noblesse en Bretagne, $X V^{e}$-XVIII siècles, Archives historiques de Bretagne, 5, 1997, p. 285-305. 
futurs doivent répondre à une série de conditions qui garantissent la validité des noces. Ces conditions sont au nombre de sept : 1- le libre consentement des contractants (c'est la substance même du mariage), 2- l'âge de puberté (14 et 12 ans accomplis pour les garçons et les filles, respectivement), 3- Le consentement des pères et mères ou tuteurs lorsque les conjoints sont mineurs (c'est-à-dire âgés de moins de 25 ans), 4- La proclamation de trois bans dans les paroisses où sont domiciliés les futurs, 5L'assistance de quatre témoins dignes de foi, 6- La bénédiction nuptiale célébrée par le curé ou le vicaire de l'un des conjoints, 7- L'absence d'empêchements (en particulier de parentés entre les conjoints) ${ }^{11}$. On le voit, ne figure dans cette liste aucune forme de restriction au libre choix des époux ou de leur famille quant à la condition sociale, quant à la nationalité et, ce qui est plus curieux, quant à la religion. Or, l'éventualité du mariage inter-religieux existe évidemment : de 500000 à 850000 protestants vécurent dans le Royaume, entre le $\mathrm{XVI}^{\mathrm{e}}$ et le XVIII ${ }^{\mathrm{e}}$ siècles, avec une liberté de conscience et un statut civil reconnu de la promulgation de l'édit de Nantes en 1598 jusqu'à sa révocation en 1685. D'autre part, une autre minorité, les juifs, subsistait en dépit des interdictions, dans des conditions, précaires en Lorraine ou en Alsace, plus enviables en Avignon ou à Bayonne et Bordeaux. Elle pouvait rassembler 40000 âmes au maximum à la fin du XVIII ${ }^{\mathrm{e}}$ siècle. Le paradoxe de cette absence de référence à la religion des époux n'est qu'apparent, puisque la proscription de la mixité religieuse se trouve en fait dans la définition même du mariage. Le mariage catholique est un sacrement. À ce titre, il ne peut concerner que deux chrétiens baptisés ${ }^{12}$ et qui, par ailleurs, reconnaissent à la cérémonie sa portée sacramentelle. Chacun sait que les réformés n'admettent que deux sacrements, ceux institués par Jésus, le baptême et l'eucharistie. De fait l'union d'un catholique et d'un protestant n'est possible qu'à la condition qu'un des deux conjoints épouse en même temps les croyances de l'autre, c'est-à-dire se convertisse ${ }^{13}$. Quant au mariage mixte unissant une famille juive à une famille chrétienne, il pose aussi beaucoup de problèmes. Aux yeux de la loi israélite, le mariage avec un non-juif n'a aucune valeur et les parents de l'enfant l'ayant contracté allaient fréquemment jusqu'à observer les rites de son deuil ${ }^{14}$.

Dans un contexte où l'agent de la célébration des noces ne peut être qu'un religieux, il va de soi que le mariage mixte interconfessionnel n'existe pas stricto sensu. Compte tenu du rapport des forces entre une religion

11. Ferriere, C.-J. de, Dictionnaire..., op. cit., art. « Mariage », p. 193-194.

12. "Ceux qui ne sont pas de notre religion ne peuvent se marier avec les Chrétiens ", Pierre Toussaint DuRAnd de MaIllane, Dictionnaire de droit canonique et de pratique bénéficiale, t. IV, article " Mariage ", Lyon, 1776, p. 35.

13. Après la Révocation de l'édit de Nantes, quand bien même tout le monde fut en principe devenu catholique, les prêtres demandaient généralement aux nouveaux convertis de se confesser et de communier avant de se marier.

14. WigODER, Geoffrey (dir.), Dictionnaire encyclopédique du judaïsme, Paris, Cerf/Robert Laffont, «Bouquins ", 1993, p. 639-640. 
d'État et des minorités au mieux à peine tolérées (l'édit de Nantes, ne l'oublions pas, interdisait le prosélytisme protestant et toute conversion d'un catholique à la Réforme), il supposait nécessairement comme préalable l'abjuration ou le baptême du non catholique. À Poitiers, le 17 juin 1777, le mariage de Jean-Baptiste Louis Augustin Isaac Rodriguès Alvarès, un juif bordelais, fut ainsi précédé de son baptême par le curé de Saint Germain, près de deux mois plus tôt ${ }^{15}$. Les origines sociales honnêtes du marrane, fils de négociant, ainsi que la rareté de ce type de conversion, à tout le moins dans une ville comme Poitiers qui n'a pas accueilli de juifs depuis un lointain Moyen Âge ${ }^{16}$, expliquent sans nul doute la qualité du parrain et de la marraine qui lui furent choisis : le marquis de Pradel, maréchal des camps et armées du roi, et la fille de l'intendant de Blossac. Il va de soi cependant, qu'en Poitou, comme d'ailleurs dans l'ensemble de la France de cette époque, le cas de figure le plus fréquent de mixité religieuse concerne les catholiques et les protestants qui cohabitent fréquemment au sein des mêmes hameaux ou des mêmes rues. C'est ainsi que, dans les campagnes du Mellois ou du Pays Mélusin, mais aussi et surtout dans les bourgs et les villes où le poids de la communauté huguenote se fait probablement moins sentir, (Loudun, Moncoutant, Civray, Thouars, etc.), il n'est pas rare qu'un ou une fidèle de la Réforme accepte une abjuration, peut-être de pure façade, pour vivre officiellement son amour avec un ou une catholique ${ }^{17}$.

Si la mixité religieuse n'existe finalement pas au regard du canoniste, que peut-on dire de ce que l'on appellerait de nos jours le mariage interethnique? À première vue, celui-ci paraît bien improbable, sauf à raisonner en dehors de l'espace territorial métropolitain. La France d'Ancien Régime, c'est aussi un ensemble de possessions, essentiellement américaines, où se rencontrent, côtoyant les colons, les soldats et les administrateurs français, des populations autochtones et des esclaves africains. Les historiens de la Nouvelle France ont établi que la mixité franco-amérindienne était relativement banale, dans les postes reculés de la traite des fourrures mais aussi en Acadie, à l'époque où la population provenant de la métropole était très majoritairement masculine. Elle ne suscitait pas d'ailleurs la réprobation de l'État, Colbert allant jusqu'à plaider pour la fusion des deux peuples et Louis XIV donnant son " entière approbation " au mariage des colons avec de jeunes Indiennes " pourvu qu'elles soient

15. Arch. dép. de la Vienne, $9^{\mathrm{E}}$ 229/30, registre de la paroisse Saint Germain. Le baptême fut célébré le 27 avril 1777.

16 Les Juifs furent expulsés du Poitou et de la Saintonge à la fin du XIII e siècle.

17. C'est par exemple le cas de Jacques de Guillon, seigneur des Varennes, "nouveau converti à la Religion catholique " que le curé d'Availles-Limouzine marie avec sa parente Esther d'Argence, elle aussi cependant de récente souche huguenote (mairie d'Availles, registre paroissial, acte du 21 juin 1682). En l'espèce, l'abjuration vise d'abord à garantir, en période de répression de la Religion réformée, une stratégie matrimoniale de repli : la mixité n'est qu'apparente et dissimule une forte homogamie (identité de la souche familiale et de la tradition religieuse). 
chrétiennes $^{18}$ ». Dans une société où le référent identitaire premier est la religion, on pourrait penser que le mariage entre Blancs et Africains christianisés n'aurait pas posé plus de difficultés. C'était sans compter avec le statut juridique particulier qu'est celui de l'esclave, une position infrahumaine qui exacerbe la distance sociale et du coup les préventions vis-àvis du mariage inégal. Depuis le Code Noir de 1685, l'esclave est, en effet, à la fois un bien mobilier et un être humain sans droit. Pour autant, le mariage avec une personne libre ne lui est pas interdit mais suppose automatiquement l'affranchissement ${ }^{19}$. Dans ce contexte-là, il est clair que c'est moins l'origine ethnique de l'esclave qui pose problème que son statut social : la mixité relève ici plutôt d'une hétérogamie extrême que l'union annule au moins dans sa dimension juridique. Cette réglementation a pris ensuite une tournure plus nettement raciste avec le Code Noir de Louisiane de 1724 dont l'article 6 défend aux " sujets blancs de l'un et l'autre sexe de contracter mariage avec les Noirs " comme " de vivre en concubinage avec des esclaves ${ }^{20}$ ". Dès 1713, par crainte que la Louisiane ne devienne une colonie de mulâtres, "naturellement fainéants, libertins et encore plus fripons ", le commissaire ordonnateur Duclos y avait condamné "l'altération que de pareils mariages feront à la blancheur, et à la pureté de sang dans les enfants ${ }^{21}$ ". Cette société coloniale qui ne dissimule pas son mépris pour l'Africain asservi n'avait peut-être pas encore exporté ses " valeurs " jusqu'à Pleumartin, en Poitou, où un mariage mixte de ce type a été célébré en 1733. Les époux, Pierre, " nègre américain " et Louise Deslandes se sont sans doute rapprochés à la faveur de leur condition de domestiques dans la maison du marquis de Pleumartin ${ }^{22}$. Le dénommé Pierre n'était évidemment plus un esclave puisque cette forme d'aliénation était interdite en métropole : ses noces, certes mixtes selon nos critères, mais finalement homogames, ne lui firent donc au final apparemment pas gagner grandchose en terme de reconnaissance sociale ${ }^{23}$. La tolérance à l'égard des

18. HaVARD, Gilles et Vidal, Cécile, Histoire de l'Amérique française, Paris, Flammarion, 2003, p. 246.

19. L'article 9 punit les hommes libres et les maîtres ayant eu des enfants avec leurs concubines esclaves, mais précise que cette peine ne vaut pas «lorsque l'homme libre qui n'était point marié à une autre personne durant son concubinage avec son esclave, épousera dans les formes observées par l'esclave sadite esclave, qui sera affranchie par ce moyen et les enfants rendus libres et légitimes " (SALA-Molins, Louis, Le Code Noir ou le calvaire de Canaan, Paris, PUF, 1987, p. 108-109).

20. Ibidem.

21. Citations tirées de HaVARD, G. et Vidal, C., Histoire de l'Amérique française, op. cit., p. 246-247.

22. Mairie de Pleumartin, registre paroissial, 17 février 1733. Le mariage est célébré en présence du marquis et de la marquise de Pleumartin, de Françoise de la Roche Posay, du chevalier de Voulon et du marquis de la Roche Posay.

23. Le statut de chef de famille le plaçant en position de fonder un lignage et de transmettre des appartenances et des biens, changea pourtant du même coup l'expression de son identité. Celle-ci, d'un simple prénom sur l'acte de mariage, signe de son appartenance à la communauté chrétienne et de son droit au sacrement matrimonial, se mua en "Pierre Hibou " au baptême de son premier enfant. Tout se passe comme si la 
quelques unions inter-raciales célébrées en France devait cependant bientôt se heurter à la phobie naissante de la dégénérescence de la race ${ }^{24}$ : la législation en métropole se mettait au diapason des mesures en vigueur dans les colonies avec l'arrêt du Conseil d'État du 5 avril 1778 "portant défense de célébrer mariage entre les Blancs, Noirs, mulâtres et autres gens de couleur et à tous notaires de passer aucun contrat entre eux ${ }^{25}$ ".

\section{Le mariage avec les étrangers}

Dernière forme de mixité matrimoniale répondant à la définition contemporaine, le mariage entre Français et étrangers suppose que l'on ait délimité clairement les contours de ces deux notions. Qu'est-ce qu'un étranger sous l'Ancien Régime? À cette question, un Dictionnaire comme celui de Furetière répond par une formule que ne démentiraient guère ses successeurs du XxI ${ }^{\mathrm{e}}$ siècle : l'étranger est celui " qui est né en un autre pays ${ }^{26}$ ". Il existait, en outre, durant la période monarchique, une conception juridique de la nationalité et un statut de droit commun à tous les sujets du roi de France. Ces dispositions impliquaient, du même coup, des incapacités pour tous ceux qui n'étaient pas considérés comme sujets du souverain Très Chrétien. Aussi, à la distinction Français/étrangers, préférera-t-on celle en vigueur à l'époque, séparant, du point de vue du droit, le régnicole de l'aubain. Le régnicole est donc un sujet de la couronne, une personne née et résidant dans le royaume, c'est-à-dire sur une terre soumise à l'obéissance du roi de France (ce qui inclut les colonies). Par voie de conséquence, est aubain tout individu qui n'est pas né dans cet espace, quand bien même il serait d'origine française ${ }^{27}$. Celui-ci, dès lors qu'il réside en France, est

construction de la cellule familiale conférait avec ce surnom à l'ancien esclave une existence " complète ", à part entière, ajoutant une extension héréditaire à son identité individuelle. Notons, en outre que les captifs africains recevaient sur les navires négriers un nom tiré de l'histoire ancienne ou de la mythologie. Dans les plantations, un sobriquet soulignant un trait physique ou l'ethnie d'origine venait s'y ajouter ou s'y substituer. Avec le baptême, apparaissent ensuite les prénoms chrétiens qui s'imposent généralement comme noms officiels. Aucune de ces dénominations n'était cependant en usage entre les esclaves qui continuaient à s'appeler entre eux des noms africains portés avant la déportation (DEBIEN, Gabriel, Les esclaves aux Antilles françaises, XVII ${ }^{e}$-XVIII ${ }^{e}$ siècles, BasseTerre, Fort-de-France, 1974, p. 71-73).

24. Sur ce thème, voir DuchET, Michèle, Anthropologie et histoire au siècle des Lumières, Paris, Maspéro, 1971.

25. ISAMBERT, JOURDAN, DECRUSY, Recueil Général des Anciennes lois françaises depuis l'an 420 jusqu'à la Révolution de 1789, Paris, 1826, tome 25 (10 mai 1777-31 décembre 1778), p. 257-258. Cette mesure fait suite à la déclaration du roi du 9 août 1777 interdisant l'introduction de noirs, mulâtres et gens de couleur dans le royaume. La Révolution supprima cette disposition qui fut néanmoins rétablie sous Bonaparte par arrêté du 8 janvier 1803 .

26. Furetiere, A., Dictionnaire universel..., op. cit, t. 1, art. "Estranger ", p. 1029. L'article relève par ailleurs que « les Suisses, les Savoyards en France ne sont point réputez estrangers".

27. SAHLINS, Peter, "La nationalité avant la lettre. Les pratiques de naturalisation en France sous l'Ancien Régime ", Annales HSS, septembre-octobre 2000; n 5, p. 1081-1108. 
frappé de certaines incapacités juridiques, principalement deux : celle, d'abord qui l'empêche de tester et de transmettre à ses héritiers ses biens qui sont dès lors confisqués par le roi en vertu du fameux droit d'aubaine; celle, ensuite qui lui interdit d'acquérir et d'exercer un office ou un bénéfice. Pour obtenir les mêmes droits que le régnicole, l'aubain n'avait d'autre solution que de demander des lettres de naturalité à la Chancellerie. Une telle requête, systématiquement satisfaite, était très souvent formulée lorsqu'un ressortissant étranger envisageait d'épouser un sujet régnicole et de rester vivre en France. Pour une raison bien évidente : la fonction première et officielle du mariage étant la procréation, il était dans l'intérêt du conjoint étranger de faire en sorte que sa progéniture puisse jouir de son patrimoine. C'est ainsi, par exemple, que Valentin Keating, originaire d'Irlande, a demandé des lettres de naturalité pour toute sa famille en 1777, avant de marier ses filles à Poitiers et de faire valoir dans leur contrat notarié ses promesses successorales ${ }^{28}$. Comme, dans le cas des mariages interconfessionnels, nous sommes de nouveau confrontés là à une mixité de fait annulée en droit par une procédure préalable d'harmonisation.

Cela étant dit, une fois que l'on a distingué le régnicole de l'aubain, on n'a pas pour autant épuisé la réalité de ce que recouvre la notion d'étranger dans les sociétés d'Ancien Régime. Il est clair que les frontières du royaume ne suffisent pas à définir l'extranéité, dans la mesure où elles se superposent évidemment fort mal à ce qu'on pourrait appeler des " frontières mentales ". " Celui qui n'est pas du pays ", n'est, en effet, pas forcément un ressortissant d'un autre État. L'étranger, ce peut être en premier lieu celui qui parle une langue différente. On sait que la France est traversée par la ligne qui sépare les parlers d'oïl des dialectes occitans : le sentiment de dépaysement que l'idiome incompréhensible du Limousin produit sur Jean de la Fontaine au cours de son séjour dans cette province ${ }^{29}$ fut sans doute partagé par nombre d'autres septentrionaux en voyage dans le Midi. De ce point de vue, il n'est sans doute pas absurde de considérer a priori comme mixte le mariage unissant en 1685 à Poitiers, le menuisier Jean Roquechot, venu " de la province d'Agen ", avec la fille d'un marchand de vieille souche locale ${ }^{30}$. En revanche, l'effet " frontière " ne semble pas spécialement orienter les choix matrimoniaux des Poitevins situés sur les confins de leur province, au contact des territoires influencés par les parlers occitans et soumis au droit écrit (la Basse Marche, et spécialement la sénéchaussée de Bellac), sans doute parce que les ruptures entre les pra-

28. Ces lettres furent obtenues le 29 avril 1778 (Archives nationales, 0/1/236), voir NERAUDEAU, Alexandra, Les Keating : histoire d'une famille irlandaise dans le Poitou de la fin du XVIII siècle, mémoire de maîtrise sous la direction de S. Jahan, Poitiers, 1999, p. $37-41$.

29. "Passé Chavigni (Chauvigny), l'on ne parle quasi plus français " (Jean de la Fontaine, Relation d'un voyage de Paris en Limousin, lettre à Madame de la Fontaine, 19 septembre 1663, dans Euvres complètes, II, Paris, Gallimard, "La Pléiade », 1958, p. 567).

30. Médiathèque François-Mitterrand, Poitiers, registre de la paroisse Saint-Savin, Mariage Jean Roquechot-Anne Parchandier, 14 novembre 1685. 
tiques et le langage sont atténuées par le contact séculaire et une démarcation moins linéaire qu'en dégradés et en transitions douces ${ }^{31}$.

Plus strictement encore, l'étranger, indépendamment même de tout référent linguistique, pourrait être aussi celui qui n'appartient pas à la communauté. En milieu rural, spécialement, des historiens de la culture populaire comme Natalie Zemon Davis ou Robert Muchembled ont étudié ces rituels de la jeunesse masculine dont le but était de contrôler le marché matrimonial de leur paroisse. Les garçons, organisés en bandes, s'efforçaient d'interdire ou du moins de limiter l'accès des célibataires des paroisses alentours à leur "stock " de jeunes filles à marier. L'existence de ce que Robert Muchembled appelle une " xénophobie de proximité " reste à prouver ${ }^{32}$. À tout le moins, elle ne nous paraît pas généralisable à toutes les zones géographiques. Des monographies concernant certaines régions du Poitou, comme la région de l'Isle-Jourdain, par exemple, tendent à établir que les solidarités étaient fortes entre des populations de communautés limitrophes, habituées à pratiquer ensemble la migration saisonnière des métives en Loudunais ou en Mirebalais et appelées à changer fréquemment de domicile, d'une paroisse à une autre, au gré des baux de colonage $^{33}$. La relative fréquence des mariages entre habitants de paroisses distinctes, même séparées par une rivière ${ }^{34}$ ou par une frontière provinciale témoigne en outre du caractère peu efficace ou du moins purement symbolique de cet esprit de clocher, nous autorisant du même coup à ne pas considérer comme " mixtes" des unions qui ne sont qu'exogames.

Pour l'époque qui nous intéresse, et dans l'optique d'une exploration des formes de mixité, la question se pose donc de savoir où finit le semblable et où commence l'autre. Les frontières territoriales d'un "pays ", d'une province, d'un royaume suffisent-elles à circonscrire ces catégories? D'évidence non. Peut-être bien plus objet de la méfiance que l'individu qui vient d'un endroit lointain mais déterminé, serait l'individu qui arrive de nulle part. Il existe en effet une sorte d'extraterritorialité propre à l'étranger de l'" intérieur ", de celui qui, pour être sujet régnicole et pour parler le même langage, n'en apparaît pas moins " autre ", sans que cette altérité s'enracine dans un espace donné et s'identifie à une origine. Ces frontières

31. La zone dont on parle - les confins poitevins de la Basse-Marche - est marquée par des parlers occitans à affinité d'oïl.

32. MuchEMBLED, Robert, L'Invention de l'homme moderne. Culture et sensibilités en France au XVIIT siècle, Paris, Fayard, 1988, p. 309.

33. Sur les migrations saisonnières, voir JAHAN, S., " La route des métives. Les travailleurs saisonniers des confins marchois en Loudunais (XVII ${ }^{\mathrm{e}}-\mathrm{XVIII}{ }^{\mathrm{e}}$ siècles) ", dans Bulletin du Groupes de Recherche Historique et Archéologique de l'Isle-Jourdain, n 11, 2001, p. 29-42.

34. Le taux d'exogamie des paroisses de Queaux, Gouex, Persac et Moussac, en val de Vienne, est d'environ $25 \%$ pour les homes et $20 \%$ pour les femmes dans la seconde moitié du XVIII ${ }^{\mathrm{e}}$ siècle. Pour les habitants de Queaux, la Vienne, ne constitue nullement un obstacle, puisque la majeure partie des mariages exogames se font dans les paroisses situées de l'autre de la rivière, accessibles seulement grâce au passeur (Sébastien GIRARD, Le Val de Vienne aux XVII et XIX ${ }^{e}$ siècles. Une étude démographique de quatre paroisses, mémoire de maîtrise, Poitiers, sans date, p. 27 et annexes). 
invisibles sont, par exemple, celles qui séparent, aux XVII ${ }^{\mathrm{e}}$ et XVIII ${ }^{\mathrm{e}}$ siècles, les populations nomades de la masse des sédentaires. Les ouvriers de la métallurgie et ceux de la sylviculture qui circulaient alors en groupe avec toute leur famille, de forge en forge ou de forêt en forêt, sans être véritablement victimes du rejet des habitants où ils s'installaient, trouvaient assez rarement un parti en dehors de leur propre milieu ${ }^{35}$. Il y a plusieurs explications à cela. On pense bien sûr avant tout à la suspicion qui pèse alors sur toute personne "sans aveu ", spécialement pour les forestiers qui vivent dans d'humbles cahutes de lisière, en marge du terroir et dont la présence ne dure que le temps du chantier. Privé de tout enracinement immobilier, l'ouvrier itinérant n'a en outre pas grand-chose à offrir en terme de dotation matrimoniale à une famille du terroir. Bien plus essentielles me semblent être les incompatibilités des cycles et des modes de vie, partant les compromis importants que supposerait toute union entre les deux groupes de population. Pour les paysans sédentaires, il va de soi que donner sa fille à un charbonnier nomade, se condamnant ainsi à ne plus la revoir, relève du sacrifice. Inversement et par voie de conséquence, pour les forestiers, lâcher une fille à un homme du finage, supposerait se priver d'un bien précieux, nécessaire à la survie d'un milieu voué à la mobilité permanente, au sein duquel le couple reste le seul référent stable d'un chantier à l'autre. Ce sont toutes ces difficultés qui justifient, à mon sens, qu'un mariage entre un nomade et un sédentaire, tous deux catholiques, français, parlant des patois semblables ou approchants, peut être qualifié de mixte.

L'historien ne saurait donc se contenter de plaquer les catégories forgées par les sociologues du xx $x^{\mathrm{e}}$ siècle sur la réalité qu'il observe, sans les repenser à l'aune de l'expérience et des représentations des acteurs du passé. Hier autant qu'aujourd'hui, l'identité ne se laisse pas figer dans un cercle unique et précisément circonscrit. Il convient particulièrement de se défier des distinctions toutes faites comme catholiques/protestants ou aubains/régnicoles qui correspondent, certes, à des fractures juridiques fortes mais peuvent aussi déformer le contenu de l'expérience vécue par les individus. L'imaginaire des guerres de religion, par exemple, a tendance à faire passer pour plus antagoniste qu'elle ne l'est vraiment la cohabitation des confessions. Surtout, l'idée d'une mixité culturelle associée au mariage entre un Français et un ressortissant étranger doit être réévaluée à l'aune des distances sociales d'autrefois. Au XVIII ${ }^{\mathrm{e}}$ siècle, l'écart - en matière de savoir, de langage, de mode de vie et de vision du monde - entre un paysan exploitant sa petite métairie et un grand bourgeois, officier de justice ou négociant de la ville voisine est bien plus large qu'entre un noble irlandais et son semblable français. L'un comme l'autre, ces derniers parlent couramment le français, langue de l'élite européenne, ont lu les mêmes livres, partagent généralement la même foi et les mêmes codes de comportement en société, etc.

35. JAHAN, Sébastien et DiON, Emmanuel, Le Peuple de la forêt. Nomadisme ouvrier et identités dans la France du Centre-Ouest aux Temps modernes, Rennes, PUR, 2002, p. 114-117. 


\section{Les sources : un apport limité}

La dernière grande difficulté qui se présente à l'enquêteur, est le repérage de ces mariages mixtes, dans la mesure où aucun document de l'époque moderne n'a eu spécifiquement vocation à les recenser comme tels. La recherche se fonde donc, comme souvent en histoire, sur des traces involontaires, éparpillées dans ces séries classiques de l'enregistrement matrimonial que sont les registres paroissiaux et les minutiers notariaux. Les contrats de mariage sont malheureusement, au moins en Poitou, trop laconiques sur l'origine des conjoints et ne sauraient constituer le champ de recherche initial. À l'inverse, dans son registre paroissial, le curé, s'il ne mentionne pas systématiquement le lieu de naissance, ne se prive souvent pas de mettre en évidence la différence et le particulier. Le protestant qui a abjuré récemment se verra qualifié de "nouveau converti ", le migrant lointain sera identifié par sa province ou son diocèse d'origine, ou, s'il y a lieu, par l'indication de sa " nation " d'appartenance. Ce terme, rappelonsle, n'est pas forcément assimilable à ce que nous appelons la nationalité, mais s'apparente plutôt à une caractérisation ethnico-culturelle ou linguistique sans dimension politique nécessaire : c'est ainsi que l'on parle au XVIII siècle de "nation allemande " pour des Prussiens comme pour des Rhénans, mais aussi de "nation juive ", de "nation bohème ou égyptienne " pour les Tziganes, ou encore d'un "nègre de nation ".

Le plus souvent, cependant, ces indications n'existent pas et le mariage mixte ne peut du coup être débusqué si l'identité caractéristique des conjoints n'a pas été définie au préalable. Le problème est particulièrement délicat pour la majeure partie de la population protestante française, poussée à la conversion par les dragonnades ou par la perte de son statut civil après la révocation de l'Édit de Nantes en 1685. S'il n'a pas les moyens de se rattacher à une église du Désert, le huguenot est alors contraint de faire enregistrer son mariage et le baptême de ses enfants parmi la masse des actes de catholicité. Si le curé ne le signale pas comme "nouveau converti", rien ne viendra plus le distinguer, hormis le port de prénoms bibliques (David, Abel, Jérémie, Esther, Suzanne, etc.) qui n'a de toute façon rien d'exclusif. Toute enquête sur les mariages mixtes dans la génération qui a suivi la phase de répression optimale (1681-1685) doit donc s'appuyer sur la reconstitution des familles protestantes d'un endroit donné pour pouvoir ensuite suivre le cours des destinées individuelles. La brève étude que nous avons entreprise sur la petite communauté isolée du Vigeant a permis ainsi de reconnaître comme mixtes bon nombre des mariages de notables célébrés entre 1695 et $1709^{36}$. Les enfants des Brun ou Gardien, qui ont dû abjurer leur foi calviniste dix à trente ans auparavant, se sont ainsi fondus dans la masse de la population locale ${ }^{37}$. Restait à apprécier vraiment le degré de

36. JAHAN, S., " Jalons pour l'histoire de la communauté protestante du Vigeant, XVI ${ }^{\mathrm{e}}$ XVIII ${ }^{\mathrm{e}}$ siècles ", dans Bulletins du GRHAIJ, n $^{\circ}$ 8, 1998, p. 61-63.

37. Jacques Marcadé considère pareillement que la disparition du protestantisme minoritaire dans certaines paroisses du Poitou est liée à la fusion « dans la religion dominante 
mixité de ces unions intervenues longtemps après le traumatisme de la persécution et, parfois, au terme d'un processus de rééducation des jeunes âmes par le clergé catholique. On mesure ainsi sa chance, lorsqu'on dispose d'une lettre du curé évoquant à l'intendant l'assiduité à la messe de ses paroissiens nouvellement convertis et de leur famille ${ }^{38}$. C'est le cas au Vigeant, où il est remarquable de constater que le mariage avec des catholiques s'est opéré sans difficulté et dans le respect d'une apparente homogamie sociale pour tous les membres de la famille Gardien, devenus, des " exemples de vertu ". En revanche, chez les Brun, visiblement beaucoup plus tièdes à l'égard de leur nouvelle religion, on ne s'est résolu à l'intermariage qu'à contrecoeur ou dans la douleur, peut-être même sous la pression du clergé local ${ }^{39}$. L'une des filles de l'apothicaire Isaïe Brun s'est mariée à un humble aubergiste qui ne sait pas écrire, la plus jeune et la plus acharnée a fini par épouser un chirurgien catholique, mais à l'âge tardif de 28 ans et après la mort de son père... Évidemment, ces deux dernières unions relèvent beaucoup plus de la mixité que celles de protestants mués en «bons catholiques".

S'agissant cette fois-ci du mariage avec un étranger ou une étrangère, sa visibilité, en l'absence de toute mention d'origine, n'en est pas moins facilitée par l'onomastique. La chasse au patronyme " exotique " est à cette époque beaucoup moins aléatoire que de nos jours, compte tenu de la relative stabilité des lignages qui rend automatiquement détectable toute apparition d'un sang nouveau ou tout isolat généalogique dans le registre où sont répertoriés les rythmes vitaux de la communauté. Elle peut toutefois être perturbée par deux impondérables. Il faut ainsi compter avec la tendance de certains curés à franciser les noms étrangers, comme ce fut le cas à Monthoiron, près de Châtellerault pour ce colon rhénan nommé De Klercq rebaptisé d'un "Leclerc " beaucoup plus passe-partout ${ }^{40}$. Tout aussi piégés peuvent être les noms à consonances francophone, portés par des aubains (comme ce Sébastien Dupont, toujours à Monthoiron, en fait de nation " allemande " parce que natif de la principauté de Liège) ou, au contraire les patronymes d'allure étrangère mais arborés par des régnicoles (des Alsaciens, par exemple). L'autre difficulté propre à la recherche

à la suite de mariages mixtes " ou bien au départ des réformés "vers des zones où leurs coreligionnaires étaient majoritaires " (Les protestants poitevins de la Révocation à la Révolution, La Crèche, Geste éditions, 1998, p. 140-141).

38. Arch. dép. de la Vienne, C 55 : lettre du 16 octobre 1702 du prieur curé Bouthier à l'intendant de la Généralité de Poitiers.

39. Au début du XVIII ${ }^{\mathrm{e}}$ siècle, le clergé catholique s'efforçait d'encourager les mariages mixtes pour accélérer la dissolution du protestantisme. On sait que le missionnaire irlandais Thomas Gould (1657-1734), attaché à la " conversion des prétendus réformés du Poitou " recommandait dans sa méthode d'interdire aux nouveaux convertis de se marier entre eux (Krumenacker, Yves, Les Protestants du Poitou au XVII e siècle, 1681-1789, Paris, Honoré Champion, 1998, p. 198-199).

40. JAHAN, S., "Les Allemands du Marquis. Immigration et intégration dans les brandes du Châtelleraudais au XVIII ${ }^{\mathrm{e}}$ siècle ", Revue d'Histoire du Pays Châtelleraudais, $\mathrm{n}^{\circ}$ 5, $1^{\mathrm{er}}$ semestre 2003, p. 4-27. 
du mariage mixte " international ", c'est qu'il ne concerne que des petits nombres. Dans une région non frontalière, davantage zone de passage que terre d'accueil, comme le Poitou, la mesure statistique du phénomène n'est pas concevable. On sait, par avance que les quantités seront négligeables. De fait, l'étude est orientée selon des préoccupations essentiellement qualitatives. Il est intéressant dans un premier temps de s'interroger, une fois jaugé autant que faire se peut le degré de la mixité, sur la motivation des conjoints. Du point de vue de l'immigrant, cela revient à se demander si son choix répond, au-delà de considérations affectives évidemment non négligeables mais difficilement perceptibles, à des objectifs d'intégration ou de promotion sociale. C'est ainsi que les colons rhénans de Monthoiron ont réussi à se faire une place sur ces terres de brandes qu'ils étaient venues défricher et à s'émanciper de la tutelle de leur protecteur, le marquis agronome de Pérusse des Cars : le mariage dans les familles paysannes du cru équivalait à une " adoption ", les connectant à un réseau familial utilisé en particulier pour accéder au marché des fermes et des mutations immobilières ${ }^{41}$. Envisagé à partir de la société autochtone et du groupe familial qui reçoit, le mariage - outre l'attrait de la différence - peut aussi se comprendre comme l'issue d'une stratégie d'accès à des ressources manquantes ou alternatives. C'est ce qui se produit dans ce que les sociologues américains appellent les " compensatory intermarriage ". Un " échange compensatoire réciproque " rapproche alors l'étranger d'un lignage du cru sur la base d'une convergence d'intérêts. Si les filles de l'Irlandais Valentin Keating, dont nous avons déjà parlé, trouvent plus facilement un parti que ses fils, c'est certes en raison de pratiques nobiliaires qui redoutent peu la mésalliance des garçons et conçoivent, au contraire, l'union des filles comme une opération de prestige : vu du côté poitevin, il n'est pas grave que l'homme épouse une étrangère à la noblesse quelque peu douteuse, alors que transformer une héritière bien née en Madame Keating passait moins inaperçu. Mais, d'un autre côté, outre leur possible charme exotique, les demoiselles Keating apportaient à de vieilles familles endettées comme celle du comte d'Orfeuille une dot en espèces sonnantes et trébuchantes bien commode ${ }^{42}$. Les termes de l'échange matrimonial compensatoire tenaient dans ce marché scellé par le contrat de mariage : le crédit symbolique du nom, contre celui, plus tangible, du numéraire. Toujours perçu avec les yeux de l'autochtone, mais cette fois en dehors de l'élite, le mariage avec un étranger peut aussi fonctionner comme un mode de délestage de filles incasables, à la réputation quelque peu entachée ou bien comme une façon d'alléger la masse successorale lorsqu'une fille est, par exemple, offerte à un forestier qui l'entraîne dans sa vie nomade.

41. Ibidem.

42. JAHAN, S., "An Irish family between assimilation and Revolution. The Keatings of Poitiers, 1777-1795 " dans O'CONNOR, Thomas et Lyons, Mary Ann (éd.), Irish Migrants in Europe after Kinsale, 1602-1820, Dublin, Four Court Press, 2003, p. 155. 
L'autre tâche, bien sûr fondamentale mais au combien délicate, consiste à définir en quoi le mariage mixte serait le signe de l'existence d'aires " d'apesanteur sociale " dans un contexte donné, c'est-à-dire de marges de liberté, non seulement pour le migrant mais aussi, ce qui est plus intéressant et moins évident $a$ priori, pour le Français (ou Poitevin) de souche. Le mariage mixte permet l'exploration d'un territoire où s'exprime de manière privilégiée la contestation du modèle, l'autonomie, même relative, du choix individuel. Cette dimension-là peut, à la rigueur, s'estimer à partir des contrats de mariage qui vont fournir deux types d'indications-clés. Il s'agit d'abord de comparer le niveau de fortune des parties et d'examiner les termes de l'échange stipulés par l'acte. Mais, surtout, à travers le nombre et la qualité des témoins, la reconstitution du réseau de relations permet de mesurer le degré d'isolement ou d'enracinement de chaque conjoint, la publicité ou non des noces ainsi que la qualité des relations sociales et des protections. Dans le cas de l'union de Rodrigues-Alvares, le Juif bordelais converti, avec une Poitevine, fille d'un menuisier décédé, en 1777, il est ainsi remarquable que le baptême seul semble avoir fait événement : les noces se déroulèrent en présence de quelques parents et amis, tandis que le contrat de mariage était signé la veille dans le secret de l'étude avec pour toutes signatures, celles des futurs époux, de la mère de la fille et du notaire ${ }^{43}$. La quasi clandestinité du mariage interroge ici sur l'opprobre qui continuerait à l'entacher, malgré la conversion. Mais de qui entendait-on se cacher? De la très conformiste et catholique société poitevine, suspecte d'antisémitisme? À moins que ne pèse aussi sur l'époux la honte d'avoir renié sa religion comme sa communauté d'origine? La médiocrité des biens et des droits déclarés par Rodrigues lors de son passage dans l'étude du notaire (" quelques effets mobiliers d'une valeur de 180 livres "), ouvre la porte à une autre explication : la conversion et la fusion dans le monde de la boutique poitevine, pourraient être aussi l'issue d'un revers de fortune quelconque, pour ce fils de négociant, amené à s'exiler de la capitale prospère du grand commerce atlantique...

L'historien sera tout autant dépourvu dans sa quête de l'expression du rapport de force culturel ou du compromis interculturel qui résultent de la mixité. Il lui est par exemple impossible, à ces époques lointaines et sauf témoignage écrit, d'étudier la transmission de la langue du parent étranger aux enfants issus du couple. Dans les cas de mariages interconfessionnels, il aurait théoriquement plus de facilité à évaluer le choix d'une religion pour leur postérité, en relevant la présence ou l'absence d'un baptême catholique. En pratique, toutefois, on a vu que le baptême préalable du futur conjoint non catholique rendait toute surprise fort peu probable : le choix de la religion au moment du mariage est logiquement celui que l'on fera, du moins officiellement, pour les enfants qui en sont issus. Les seuls indices accessibles et exploitables seraient, en fait, ceux dénotant un attachement à l'origine dans le choix d'un prénom. Une enquête de ce type ne

43. Arch. dép. de la Vienne, $4^{\mathrm{E}} 12 / 279$, contrat du 16 juin 1777. 
doit toutefois pas négliger qu'un prénom, sous l'Ancien Régime, est moins un marqueur culturel qu'un estampillage généalogique : le nom de baptême est soit l'expression de la parenté spirituelle, dans la mesure où il est donné par le parrain ou la marraine, soit la réactivation ou le prolongement de la mémoire d'un parent. Si les enfants d'un couple franco-rhénan s'appellent Christian ou Frédéric, cela ne veut pas dire nécessairement que l'un des parents cultive la nostalgie de ses racines germaniques, mais qu'il a choisi un parrain portant ce prénom-là dans sa communauté d'origine ou qu'il a voulu honorer la mémoire d'un aïeul homonyme. Plus révélatrice serait donc la volonté de " francisation " perceptible dans la mutation ononastique, lisible dans la signature : au bout de quelques années, un des Allemands de Monthoiron, Wilhelm Le Klerk, finit par se faire appeler "Guillaume ${ }^{44}$ ". La transformation peut affecter aussi le nom dans son intégralité : après son mariage avec une Française, l'ancien prisonnier de guerre espagnol qui signait " Alejandro Sanchez-Duràn " se glisse dans l'identité plus anonyme d'un Alexandre Durand ${ }^{45}$.

Les quelques linéaments d'une recherche que nous avons dessinés cidessus ont tenté de démontrer que si la notion de mariage mixte n'était pas nécessairement transposable au contexte d'Ancien Régime, vêtu de ses habits contemporains, elle n'en restait pas moins un point d'observation - certes aléatoire et fluctuant - mais, me semble-t-il pertinent, de l'évolution du niveau de répression ou d'ouverture à l'altérité des autorités civiles et religieuses, comme des mécanismes de la mobilité sociale d'Ancien Régime. La mixité matrimoniale, au-delà de sa lecture romantique, peut être envisagée comme une stratégie d'implantation, par l'accès aux ressources d'un réseau connecté au marché de la terre, ou comme un mode d'émancipation qui passe par la démonstration de la " pacification " du prisonnier de guerre ou du rebelle hérétique. Il nous parle aussi du statut de la femme, très rarement ou très difficilement concédée au migrant. Mais l'inverse est tout aussi vrai : lorsque la communauté minoritaire est suffisamment importante, les femmes sont visiblement considérées comme une espèce de capital à usage interne au sein d'une économie matrimoniale étroitement contrôlée par les pères ou les frères. Les exceptions n'en sont que plus passionnantes à étudier. Dans les cas où la femme du cru est " perdue " ou trop vieille, le mariage devient une planche de salut : l'étranger venant bouleverser les coordonnées d'un destin tout tracé. Plus positivement encore, la mixité peut devenir une sorte d'issue - peut-être provisoire - à la tutelle communautaire ou lignagère : en affirmant par cette union atypique et dérangeante son autonomie de choix, la femme exprimerait là sa contestation du modèle patriarcal. Mais le plus souvent, la femme reste un instrument de l'arrangement entre familles, négocié selon les termes de

44. De la même façon, le maçon Johann Christian Leideck, signe le 11 décembre 1785 un reçu " Jean Leideck " (JAHAN, S., " Les Allemands du marquis... ", art. cit., p. 20-21).

45. Archives municipales de Poitiers, État Civil, Mariage du 29 juillet 1811, et Arch. dép. de la Vienne 2U/172, justice de paix de Poitiers Nord (1814). 
l'échange compensatoire : le mariage mixte des temps modernes nous parlerait finalement plus de subordination que de libération...

Ajoutons enfin qu'entre le début et la fin de l'époque moderne, le point d'achoppement de la mixité s'est déplacé. Dans le contexte de la course à l'évangélisation des peuples païens et de la poursuite des guerres de religion, les peurs se focalisent sur la diversité confessionnelle : peu importent finalement les origines ethniques de l'Autre pourvu qu'il partage la même foi. Le XVIII ${ }^{\mathrm{e}}$ siècle voit poindre, dans les colonies, l'angoisse de la submersion des Blancs par les esclaves et les mulâtres, relayée, en métropole, par la crainte d'une dégénération de la race européenne liée au métissage. Appuyé sur les nouvelles sciences de la nature et l'idée d'une mutabilité des espèces, le cloisonnement matrimonial qui vise à préserver la blancheur originelle de l'espèce, voire à garantir le cours du progrès humain, s'installe dans le sillage d'une pensée anthropologique où le principe de l'inégalité des races tend à légitimer la prétention de l'Européen à se surpasser et à décider du destin des autres peuples.

\section{RESUME}

Notion éminemment contemporaine, la mixité matrimoniale a-t-elle du sens appliquée à des sociétés provinciales d'Ancien Régime où l'expérience de l'autre nation ou de l'autre peuple est rarissime, où l'étranger, aussi, prend souvent les traits de celui qui vient d'une province voisine? Après une tentative de définition de ce que serait le mariage mixte pour les hommes et les femmes de l'Ancien Régime, cet article envisage à partir de l'exemple poitevin les différentes formes d'unions potentiellement problématiques (interconfessionnelles, interethniques, exogames Français-étrangers...), ainsi que leur articulation avec les hiérarchies de genre et de catégories sociales. Il aboutit enfin à s'interroger sur le degré d'ouverture de la société au $\mathrm{XVIII}^{\mathrm{e}}$ siècle comme sur ses représentations de l'identité et des appartenances multiples.

\section{ABSTRACT}

Is it possible to use the present-day notion of mixed marriage for the french Ancien Régime society, at a time when the foreigner could be the one who was coming from an adjoining province? After trying to define the mixed marriage in modern France, this article considers the different forms of potentially problematical unions (interdenominational, interethnic, between French and foreigners...), and their connections with gender or social hierarchies. Finally, the article examines the opening degree of French $18^{\text {th }}$ Century society, as the representations of identities and interbreedings. 